\title{
Microchimerism in twins
}

Małgorzata Waszak ${ }^{1}$, Krystyna Cieślik, Karolina Wielgus², Ryszard Słomski , Marlena Szalata ${ }^{3}$, Marzena Skrzypczak-Zielińska ${ }^{3}$, Joanna Kempiak ${ }^{4}$, Grzegorz Bręborowicz ${ }^{4}$

1Department of Functional Anatomy, University School of Physical Education, Poznan, Poland

2Department of Biotechnology and Molecular Biology, Institute of Natural Fibres and Medicinal Plants, Poznan, Poland

Institute of Human Genetics, Polish Academy of Sciences, Poznan, Poland

4Perinatology and Gynaecology Clinic, Medical University, Poznan, Poland

Submitted: 28 February 2011

Accepted: 4 September 2011

Arch Med Sci 2013; 9, 6: 1102-1106

DOI: 10.5114/aoms.2013.39212

Copyright @ 2013 Termedia \& Banach

\section{Abstract}

Introduction: The aim of this paper was to report the occurrence of peripheral blood chimerism in newborns from bigeminal pregnancies.

Material and methods: Cord blood collected from 50 pairs of twins constituted the biological material studied. Analyses included: DNA isolation, quantitative and qualitative assessment of DNA preparations, hybridization analysis of SLS type as well as of MLS type, and analysis of microsatellite sequences with regard to polymorphisms using polymerase chain reaction.

Results: The presence of additional fragments of DNA in peripheral blood lymphocytes was found in four out of fifty pairs of monozygotic twins (8\%) at locus D7S21 (7p22, $n=3)$ and locus D12S11 (12q24.3, $n=1)$. In these cases, the presence of additional DNA fragments was also proved by analysis of microsatellite sequence polymorphisms at loci HUMPLA2A1 (pancreatic phospholipase A-2, 12q23), HUMCYARO (cytochrome P450, 15q21.1) and HUMvWF (von Willebrand factor, 12p13).

Conclusions: The results of our study confirm the occurrence of chimerism in twins and constitutes the starting point for further studies aimed at determining the clinical significance of chimerism in twins both for women and fetuses.

Key words: chimerism, mosaicism, zygosity of twins.

\section{Introduction}

In mythology, the term "chimera" pertained to a monster which appeared to be composed of fragments of various animals. In biology, it is an organism composed of cells that contain various genetic material. Are chimeras among us? What is the likelihood of such chimeras being born?

During genetic screenings, it was found that the phenomenon of chimerism (to a limited extent) occurs surprisingly often. The co-existence of two genetically different cell populations in one organism, of which one occurs in a very low number, is called microchimerism. Possible reasons for this phenomenon include somatic mutations, as well as fertilization of the egg cell and its polar body by two sperm cells. Fetomaternal microchimerism is said to be the most frequent cause. Men are host to microchimerical maternal cells, remaining from their fetal life period. Women, apart from microchimerical maternal cells, receive a new dose of fetal cells as a result of their pregnancies. The transport of fetal cells into

\author{
Corresponding author: \\ Małgorzata Waszak PhD \\ Department of Functional \\ Anatomy \\ University School \\ of Physical Education \\ 27/39 Królowej Jadwigi St \\ 61-871 Poznan, Poland \\ Phone: +486183552 26 \\ Fax: +48 618355220 \\ E-mail: malwasz@interia.pl
}


the mother's circulation is greater than the transport of the mother's cells into fetal circulation [1], thus increasing the diversification between women and men as regards the presence of microchimerical cells. The moment determining the ultimate level of transfusion of cells between the mother and baby is delivery. Natural deliveries result in increased transport of maternal cells to the fetus, while caesarean section (apart from saving the fetus) [2] intensifies the transport of fetal cells into the mother's circulation [3].

Microchimerism may also follow from processes other than the transfer of cells between the mother and the fetus. An exchange of cells also takes place between bigeminal fetuses in the uterus. Because some bigeminal fetuses decay or are absorbed before the physician notices them, microchimerism may also originate from a "spectral twin" [4].

With newer methods of artificial procreation and the possibility of genetic testing increasing, changes in views concerning the process of twin formation will probably follow. The fact that someone may be a chimera can only be detected by means of a detailed DNA test.

Studies of microchimerism will perhaps allow for standardization of terminology explaining the occurrence of chimerism and mosaicism in twins. Organisms which consist of genetically different cells, originating from one zygote, are not called chimeras, but mosaic organisms. In the literature, it has been difficult to mark a clear line between chimerism and mosaicism in studies of twins. In reference to monozygotic twins, such a distinction is even more difficult as they are formed from one zygote. Applying the definition of chimerism and mosaicism, all cases pertaining to genetic differences in monozygotic twins should be called mosaicism. However, many authors use the term chimerism when describing the transfer of cells from one twin (A) to its co-twin (B). These twins are genetically similar to each other, with twin $B$ having a few cells from twin A. The notion of mosaicism is used to describe genetic differences resulting from single mutations which occurred after the division of the zygote in the genome of one of the twins. However, it is very difficult to state whether the genetic differences took place before or after the division of the zygote.

The presence of placental anastomoses constitutes an additional problem for comparative studies of genotypes of twins from monochorionic pregnancies. These anastomoses enable the transfusion of blood and mother cells between twins, which leads to pseudomosaicism or chimerism, i.e. the transfer of mother cells of the co-twin to marrow cells during embryogenesis.

Standardizing research methods and the consideration of pregnancy histories in detail is of key significance for enabling familiarity with the issue of microchimerism. The vast array of methods used in detecting microchimerism constitutes one of the reasons for discrepancies between the results presented in the literature [5]. The first evidence of a shift of cells from an organism (mother) to an offspring during pregnancy was based on the analysis of the karyotype of male newborns, in which the presence of male mosaicism was proven [6]. Then, owing to the polymerase chain reaction (PCR) method, it was proven that cord blood cells contain the mother's DNA in about $50 \%$ of studied samples. Real time PCR based amplification of the DYS14 gene (located on chromosome $Y$ and with a permanent number of repetitions between various individuals) allows one male cell among 100000 female cells to be detected when a shift of cells from an offspring to the mother occurred during pregnancy [7]. Real time PCR reactions with the use of primers and probes specific in relation to the sequence of HLA genes of a donor allow microchimerism to be detected regardless of the sex [8].

In the course of long-term studies on the development of twins conducted by us, the results of analyses determining their zygosity indicated the presence of additional genetic material in a few pairs of monozygotic twins. Therefore, the aim of this paper was to report the occurrence of peripheral blood chimerism in newborns from bigeminal pregnancies.

\section{Material and methods}

Cord blood was collected immediately after delivery from 50 consecutive pairs of twins born at the Perinatology and Gynaecology Clinic of the Medical University of Poznan (Poland) between 2007 and 2009. It constituted the biological material for this study.

All the procedures were approved by the Local Ethics Committee of the Medical University of Poznan. Written consent was obtained from mothers qualified for the study.

Performed analyses included: 1) isolation of DNA with the use of guanidine isothiocyanate [9], 2) the qualitative and quantitative evaluation of DNA preparations [9], 3) analysis of hybridization including the simultaneous analysis of many loci (MLS) with the $(C A C)_{5}$ probe and a single locus (SLS) with many probes (MS43A and MS31) [9], and 4) analysis of micro-satellite sequences with regard to polymorphisms using PCR [10].

\section{Results}

Analysis of the zygosity of the studied group of twins performed with the application of single locus hybridization allowed, in the case of four pairs of twins, the discovery of the existence of an additional DNA fragment. Analysis of locus D7S21 (7p22) with a specific probe (MS31) indicated the presence of an additional DNA fragment in three pairs of twins (Figure 1). On the other hand, the analysis of 
locus D12S11 (12q24.3) by means of another probe (MS43A) indicated the presence of an additional DNA fragment in one baby from one of the 50 pairs of twins studied. In this case, the other twin had a correct result (Figure 1).

A

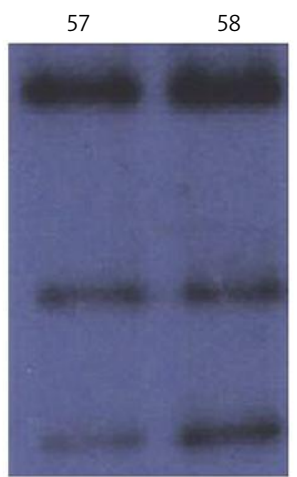

gel 1

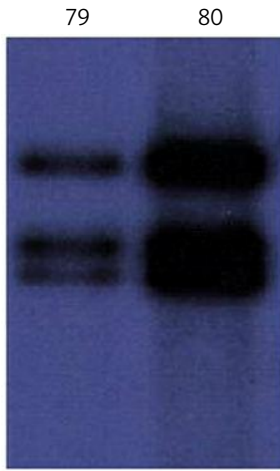

gel 2
Multilocus analysis indicated the identity (number of common alleles), which constituted the basis for confirmation of monozygosity.

The presence of additional DNA fragments in two out of the four cases described above was also

Figure 1. Demonstration of the alleged chimerism in monozygotic twins through hybridisation by means of: (A) molecular probe MS31 and (B) molecular probe MS43A. A - Monozygotic twins are characterized by the presence of identical DNA fragments. In the case of homozygosity, one DNA fragment is visible, whereas in the case of heterozygosity, two fragments are visible. Gel 1, twins from pair 57/58: three DNA fragments are visible in both babies. Gel 2, twins from pair 79/80: three DNA fragments are visible in both babies. Gel 3, twins from pair 91/92: four DNA fragments are visible in both babies. B - Results of analysis in twins from pair 35/36. Three DNA fragments are visible in baby No. 36
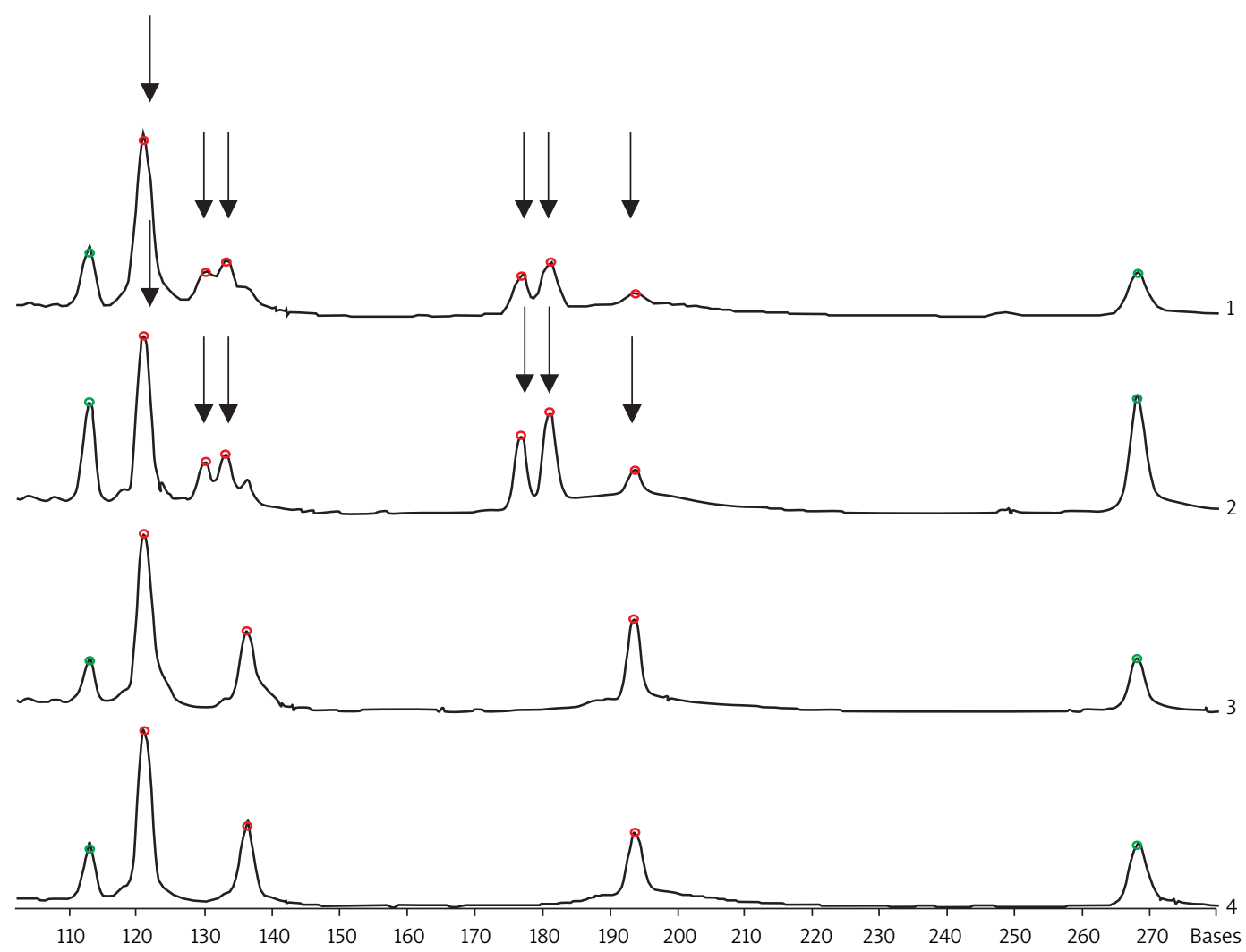

Figure 2. Demonstration of the alleged chimerism in monozygotic twins through the analysis of polymorphism of microsatellite sequences HUMPLA2A1 and HUMCYARO. On the left side of the figure, the fragments corresponding to locus HUMPLA2A1 are visible, whereas in the central part there is locus HUMCYARO. Each sample contains internal patterns with a size of 113 and 268 bp. Track 1, twin 79; track 2, twin 80; tracks 3-4, control DNA - monozygotic twins, which are heterozygotes in locus HUMPLA2A1 and homozygotes in locus HUMCYARO. In twins from pair 79/80, three alleles in locus HUMPLA2A1 and HUMCYARO are visible 

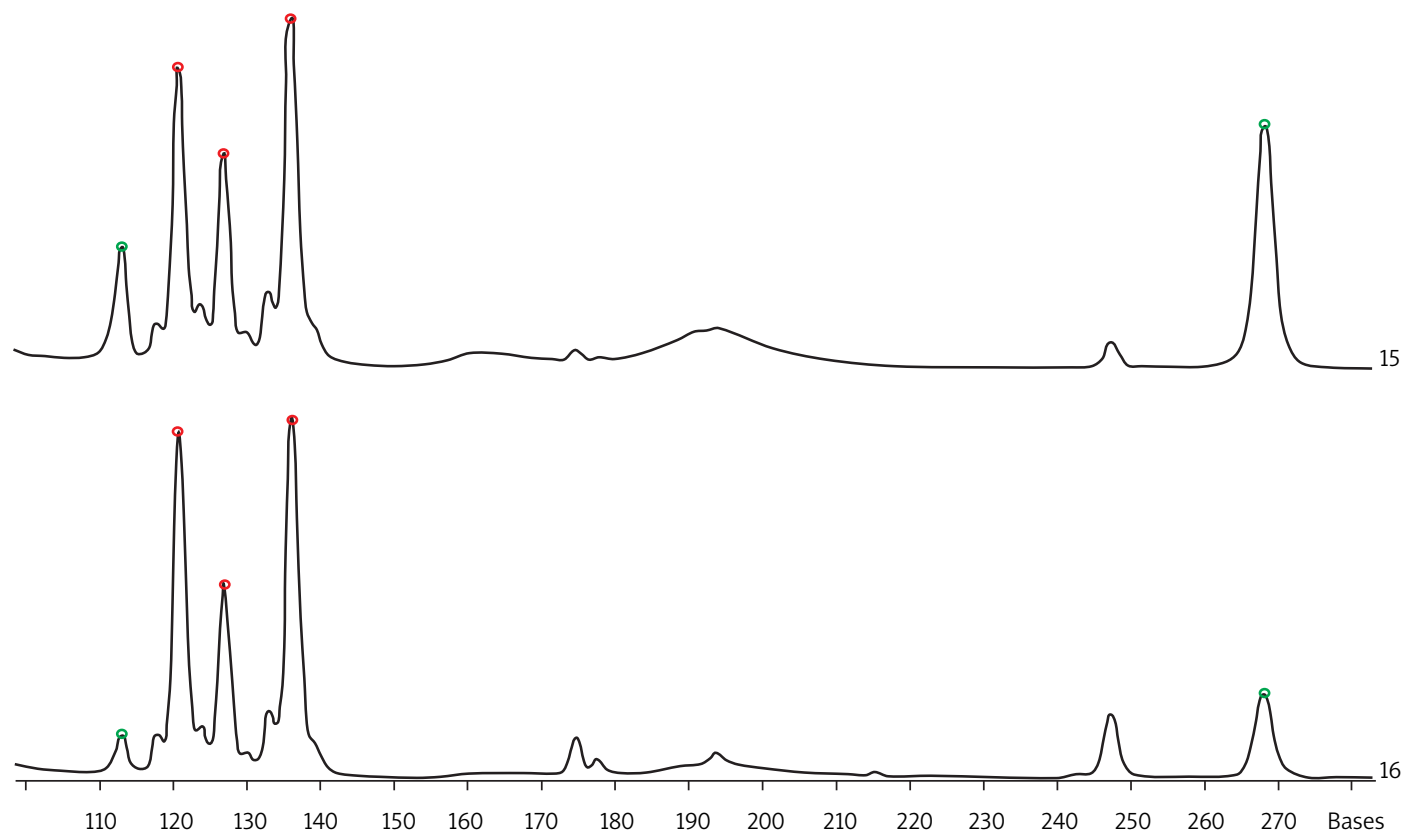

Figure 3. Demonstration of the alleged chimerism in monozygotic twins through the analysis of polymorphism of microsatellite sequence HUMPLA2A1. Alleles varying by three nucleotides were identified by determination of the size of fragments and their comparison with the allele ladder. Each sample contains internal patterns with a size of 113 and 268 bp. Track 15, twin 91; track 16, twin 92. In both twins, three alleles in locus HUMPLA2A1 are visible

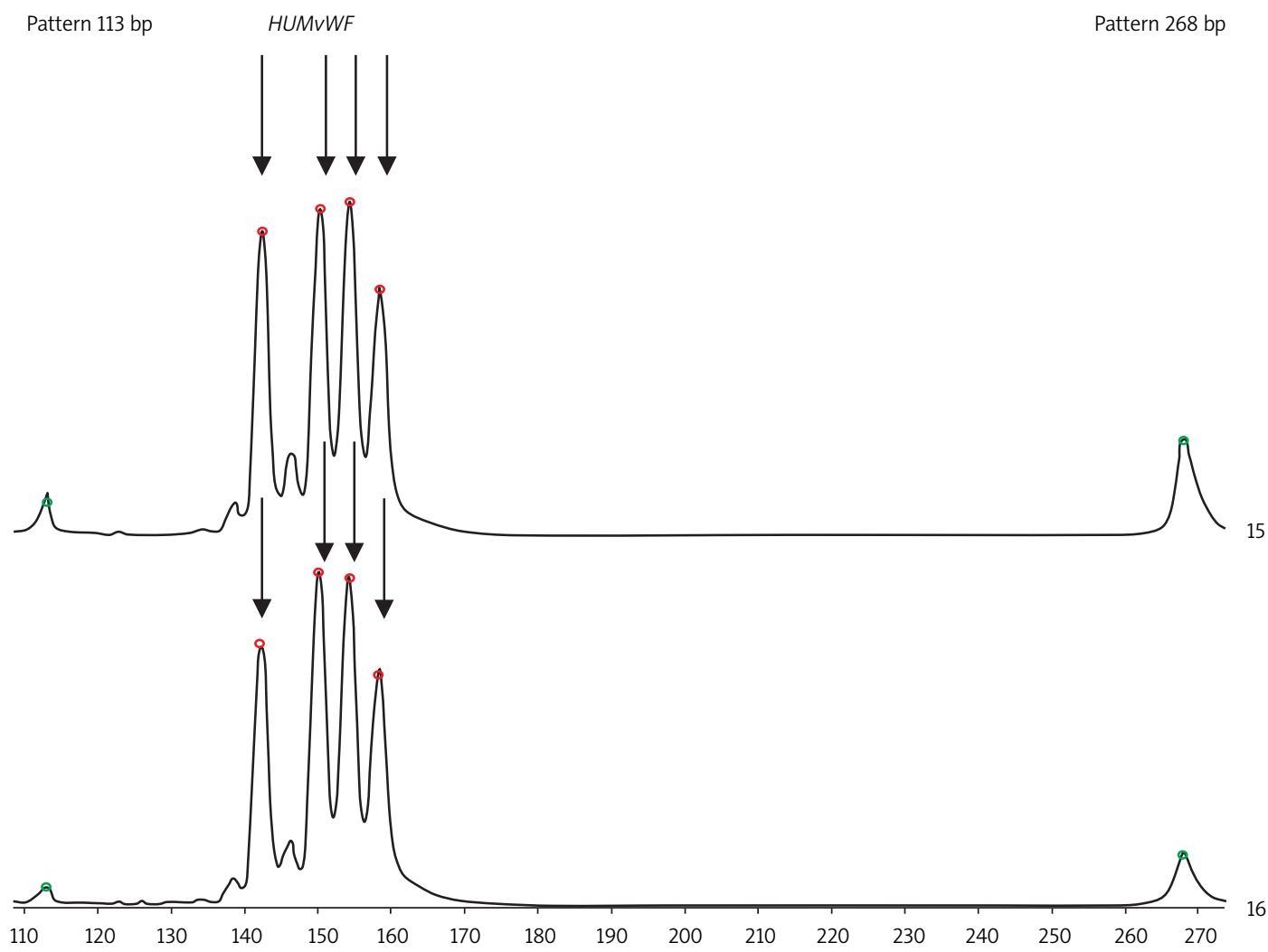

Figure 4. Demonstration of the alleged chimerism in monozygotic twins through the analysis of polymorphism of microsatellite sequence HUMvWF. Alleles varying by four nucleotides were identified by determination of the size of fragments and their comparison with the allele ladder. Each sample contains internal patterns with a size of 113 and 268 bp. Track 15, twin 91; track 16, twin 92. In both twins, four alleles in locus HUMvWF are visible 
proven by analysis of microsatellite sequence polymorphisms by application of PCR in loci HUMPLA2A1 (pancreatic phospholipase A-2, 12q23), HUMCYARO (cytochrome P450, 15q21.1) and HUMvWF (von Willebrand factor, 12p13). In pair 79/80, an additional DNA fragment was present in locus HUMPLA2A1 and in locus HUMCYARO (Figure 2). In pairs 91/92, the presence of an additional DNA fragment was discovered in locus PLA2A1. Also, two additional DNA fragments were discovered in locus HUMvWF (Figures 3 and 4).

In each of the discussed cases, the results prove significant quantitative sharing of additional DNA fragments and exclude the possibility of the material having been polluted with different DNA (resulting in a false positive).

\section{Discussion}

So far only several dozen cases of "classic" chimeras have been described in the literature, but the description of such cases among twins is a rarity [11]. The presence of additional genetic material in the cord blood of twins observed in this study can be explained by the transfusion of blood and mother cells through anastomoses present in the placenta. This has led to the occurrence of pseudomosaicism, in this case called chimerism. However, this was not the case in one pair of twins analyzed in our study, where not one but two additional DNA fragments were amplified, suggesting four alleles. This rules out a maternal transfer. Furthermore, these additional fragments were found in only one baby of the pair. The unambiguous determination of the etiology of additional genetic material present in studied pairs of twins will be possible only when molecular studies are performed on DNA isolated from tissues other than lymphocytes. These proposed studies should cover both the neonates and their parents. We are working on this at the moment.

A particularly interesting aspect regarding the presence of foreign cells, especially embryonic cells, which are weakly diversified and therefore totipotent, is their immunological impact and involvement in self-immunization processes [12-17]. At present, this is one of the possible hypotheses explaining the cause of disturbances in immunological processes. Both fetal and maternal microchimerism is a physiological phenomenon; nevertheless, in the presence of specific environmental and genetic factors, microchimeric cells may contribute to an imbalance of the immunological system in the host. Searching for the role of microchimerism certainly constitutes an important objective for clinical studies; therefore, each piece of information about its occurrence seems to be precious not only for cognitive reasons.

\section{Acknowledgments}

This paper was based on research financed by the Ministry of Science and Higher Education (project number NN303457238).

\section{References}

1. Lo YM, Lau TK, Chan LY, Leung TN, Chang AM. Quantitative analysis of the bidirectional fetomaternal transfer of nucleated cells and plasma DNA. Clin Chem 2000; 46: 1301-9.

2. Lin HH, Kao JH, Hsu HY, Mizokami M, Hirano K, Chen DS. Least microtransfusion from mother to fetus in elective cesarean delivery. Obstet Gynecol 1996; 87: 244-8.

3. Kaneda T, Shiraki K, Hirano K, Nagata I. Detection of maternofetal transfusion by placental alkaline phosphatase levels. J Pediatr 1997; 130: 730-5.

4. Yan Z, Lambert NC, Guthrie KA, et al. Male microchimerism in women without sons: quantitative assessment and correlation with pregnancy history. Am J Med 2005; 118: 899-906.

5. Rak J. Microchimerism, sex and autoimmune diseases. Kosmos 2008; 57: 19-28.

6. Turner JH, Wald N, Quinlivan WL. Cytogenetic evidence concerning possible transplacental transfer of leukocytes in pregnant women. Am J Obstet Gynecol 1966; 95: 831-3.

7. Lambert NC, Lo YM, Erickson TD, et al. Male microchimerism in healthy women and women with scleroderma: cells or circulating DNA? A quantitative answer. Blood 2002; 100: 2845-51.

8. Lambert NC, Erickson TD, Yan Z, et al. Quantification of maternal microchimerism by HLA-specific real-time polymerase chain reaction: studies of healthy women and women with scleroderma. Arthritis Rheum 2004; 50: 906-14.

9. Stomski R. Examples of DNA analyses [Polish]. Wydawnictwo Akademii Rolniczej im. Augusta Cieszkowskiego w Poznaniu, Poznan 2004.

10. Wielgus K. Determining zygotism and the analysis of mitochondrial polymorphism: twins DNA. Doctoral thesis. University of Life Sciences in Poznan, Poznan 2006.

11. Souter VL, Parisi MA, Nyholt DR, et al. A case of true hermaphroditism reveals and unusual mechanism of twinning. Hum Genet 2007; 121: 179-85.

12. Srivatsa B, Srivatsa S, Johnson KL, Samura O, Lee SL, Bianchi DW. Microchimerism of presumed fetal origin in thyroid specimens from women: a case-control study. Lancet 2001; 358: 2034-8.

13. Artlett CM, Cox LA, Ramos RC, et al. Increased microchimeric CD4+ $\mathrm{T}$ lymphocytes in peripheral blood from women with systemic sclerosis. Clin Immunol 2002; 103: 303-8.

14. Nelson JL. 2002. Microchimerism and human autoimmune diseases. Lupus 2002; 11: 651-4.

15. Renné C, Ramos Lopez E, Steimle-Grauer SA, et al. Thyroid fetal male microchimerisms in mothers with thyroid disorders: presence of Y-chromosomal immunofluorescence in thyroid-infiltrating lymphocytes is more prevalent in Hashimoto's thyroiditis and Graves' disease than in follicular adenomas. J Clin Endocrinol Metab 2004; 89: 5810-4.

16. Klintschar M, Immel UD, Kehlen A, et al. Fetal microchimerism in Hashimoto's thyroiditis: a quantitative approach. Eur J Endocrinol 2006; 154: 237-41.

17. Śliwa L. Microchimerism a potential cause of autoimmune diseases. Med Rodzinna 2009; 50: 25-8. 